\title{
TOTAL ABSOLUTE CURVATURE OF IMMERSED SUBMANIFOLDS OF SPHERES
}

\author{
By KazuYuki Enomoto
}

Let $M$ be an $m$-dimensional compact differentiable manifold and $f$ be an immersion of $M$ into $S^{m+k}$. Let $p \in S^{m+k}$ and $\pi_{p}$ be the stereographic projection from $p$ onto the tangent plane of $S^{m+k}$ at $-p$. If $p \notin f(M), \pi_{p} \cdot f$ is an immersion of $M$ into the $(m+k)$-dimensional euclidean space. Let $i$ be the inclusion of $S^{m+k}$ into $E^{m+k+1}$. In general, $\tau\left(\pi_{p} \cdot f\right)$, the total absolute curvature of $\pi_{p} \cdot f$, is not necessarily equal to $\tau(i \cdot f)$, the total absolute curvature of $i \cdot f$. If $m=1$, however, T. Banchoff showed in [1] that the average value of $\tau\left(\pi_{p} \cdot f\right)$ over all possible base points is equal to $\tau(i \cdot f)$. In this paper we generalize this theorem to immersions of $m$-dimensional compact differentiable manifolds into $S^{m+k}$.

The author wishes to express his hearty thanks to Professor T. Otsuki for his constant encouragement and valuable suggestions.

\section{Preliminaries.}

Let $M$ be an $m$-dimensional compact differentiable manifold and $F$ be an immersion of $M$ into $E^{m+k}$. Let $\nu_{F}^{1}$ be the unit normal vector bundle of $F$ with the total space $N_{F}^{1}$ and the projection $\pi: N_{F}^{1} \rightarrow M$. For any $e \in N_{F}^{1}$, we denote by $S(e)$ the second fundamental tensor of $F$ with respect to $e$. $S(e)$ is an endomorphism of $T_{\pi(e)} M$. Let $G(e)=\operatorname{det} S(e)$ and we call $G(e)$ the LipschitzKilling curvature of $F$ with respect to $e . \quad N_{F}^{1}$ is an $(m+k-1)$-dimensional orientable Riemannian manifold with the metric naturally induced from $E^{m+k}$. Let $d \mu$ be the volume element of $N_{F}^{1}$.

Set

$$
\tau(F)=\left(c_{m+k-1}\right)^{-1} \int_{N_{F}^{1}}|G(e)| d \mu(e)
$$

where $c_{m+k-1}$ is the volume of $S^{m+k-1}$.

We call $\tau(F)$ the total absolute curvature of $F$.

Let $N_{F}^{1}(x)$ be the fibre of $N_{F}^{1}$ at $x \in M . N_{F}^{1}(x)$ has the natural metric of the sphere $S^{k-1}$ and we denote its volume element by $d \sigma_{x}^{k-1}$.

Recerved April 25, 1977. 
Set

$$
\tau(F, x)=\left(c_{m+k-1}\right)^{-1} \int_{N_{F^{1}}^{1}(x)}|G(e)| d \sigma_{x}^{k-1}(e)
$$

We call $\tau(F, x)$ the absolute curvature of $F$ at $x$.

We now assume that $M$ is orientable and let $d V$ be the volume element of $M$ with respect to the metric induced from $E^{m+k}$ by $F$. Then there is a differential form $\omega$ of degree $k-1$ on $N_{F}^{1}$ such that its restriction to $N_{F}^{1}(x)$ is equal to $d \sigma_{x}^{k-1}$ and $d \mu=\omega \wedge d V$. Thus it holds that

$$
\tau(F)=\int_{M} \tau(F, x) d V(x)
$$

When $M$ is non-orientable, there is a double covering of $M, \bar{\pi}: \bar{M} \rightarrow M$ such that $M$ is orientable. Then we have $\tau(F \cdot \bar{\pi})=2 \tau(F)$.

We now consider a stereographic projection on $S^{n}$. Let $p \in S^{n}$ and $\pi_{p}$ : $S^{n}-\{p\} \rightarrow T_{-p} S^{n}$ be the stereographic projection from $p$. Then for any $q$ in $S^{n}-\{p\}, \pi_{p}(q)=p+k(q-p)$ where $k=\frac{2}{1-\langle q, p\rangle}$. Let $\left(\pi_{p}\right)_{*}: T_{q} S^{n} \rightarrow E^{n}$ be the differential of $\pi_{p}$ at $q$. Then we have the following:

Lemma 1. Let $q \in S^{n}-\{p\}$ and $X \in T_{q} S^{n}$. Then we have

$$
\left(\pi_{p}\right)_{*}(X)=k X+\frac{k^{2}}{2}\langle X, p\rangle(q-p)
$$

and

$$
\left\|\left(\pi_{p}\right)_{*}(X)\right\|=k\|X\|
$$

that $2 s, \pi_{p}$ is a conformal mapping with the scale function $k$.

Proof. Let $\sigma(t)$ be a $C^{1}$-curve in $S^{n}$ with $\sigma(0)=q$ and $\sigma^{\prime}(0)=X$. Let $k(t)$ : $=k(\sigma(t))=\frac{2}{1-\langle\sigma(t), p\rangle}$. Since $\left(\pi_{p}\right)_{*}(X)=\left(\pi_{p} \cdot \sigma\right)^{\prime}(0)$,

$$
\begin{aligned}
\left(\pi_{p}\right)_{*}(X) & =\left.\frac{d}{d t}(p+k(t)(\sigma(t)-p))\right|_{t=0} \\
& =k \sigma^{\prime}(0)+k^{\prime}(0)(q-p) \\
& =k X+\frac{k^{2}}{2}\langle X, p\rangle(q-p)
\end{aligned}
$$

Since $\langle q-p, q-p\rangle=2(1-\langle q, p\rangle)=\frac{4}{k}$ and $\langle q, X\rangle=0$,

$$
\begin{aligned}
\left\langle\left(\pi_{p}\right)_{*}(X),\left(\pi_{p}\right)_{*}(X)\right\rangle & =k^{2}\langle X, X\rangle-k^{3}\langle X, p\rangle^{2}+\frac{1}{4} k^{4}\langle X, p\rangle^{2}\langle q-p, q-p\rangle \\
& =k^{2}\langle X, X\rangle .
\end{aligned}
$$




\section{Main theorem.}

We now state the main theorem of this paper.

THEOREM. Let $M$ be an m-dimensional compact differentrable manifold and $f$ be an immersion of $M$ into $S^{m+k}$. Let $\imath$ be the inclusion of $S^{m+k}$ into $E^{m+k+1}$. Then

$$
\tau(\imath \cdot f)=\left(c_{m+k}\right)^{-1} \int_{S m+k} \tau\left(\pi_{p} \cdot f\right) d \sigma^{m+k}(p)
$$

where $d \sigma^{m+k}$ is the standard volume element of $S^{m+k}$.

Note: The function $p \mapsto \tau\left(\pi_{p} \cdot f\right)$ is defined almost everywhere in $S^{m+k}$ (in fact it is defined in $S^{m+k}-f(M)$ ) and continuous there.

We first consider the absolute curvature of $\imath \cdot f$. Let $N_{f}^{1}(x)$ be the unit normal vector space of $f$ at $x \in M$. Let $e \in N_{f}^{1}(x)$ and $a_{\imath}(e)(1 \leqq \imath \leqq m)$ be a system of principal curvatures of $f$ with respect to $e$. Let $X_{2}(e)$ be (unit) principal vectors of $f$ with respect to $e$ with the principal curvatures $a_{i}(e)$ respectively. For convenience, we use the same notation $x$ for the image of $x$ by $f, f(x)$. If $\bar{e}$ is a unit normal vector of $\imath \cdot f$ at $x, \bar{e}=x \sin \rho+e \cos \rho$ for some $e \in N_{f}^{1}(x)$ and $\rho \in\left[-\frac{\pi}{2}, \frac{\pi}{2}\right]$.

LEMMA 2. $X_{2}(e)$ are the principal vectors of $i \cdot f$ with respect to $\bar{e}$ with the principal curvatures $\sin \rho+a_{i}(e) \cos \rho$ respectively.

Proof. Let $S_{e}$ be the second fundamental tensor of $f$ with respect to $e$ and $\bar{S}_{\bar{e}}$ the one of $\imath \cdot f$ with respect to $\bar{e}$. Since $\bar{S}_{e}\left(X_{i}(e)\right)=S_{e}\left(X_{i}(e)\right)=a_{\imath}(e) X_{i}(e)$,

$$
\begin{aligned}
\bar{S}_{\bar{e}}\left(X_{\imath}(e)\right) & =\sin \rho \bar{S}_{x}\left(X_{i}(e)\right)+\cos \rho \bar{S}_{e}\left(X_{\imath}(e)\right) \\
& =\left(\sin \rho+a_{i}(e) \cos \rho\right) X_{\imath}(e)
\end{aligned}
$$

q. e. d.

Let $\bar{G}(\bar{e})$ be the Lipschitz-Killing curvature of $i \cdot f$ with respect to $\bar{e}$. Then we have $\bar{G}(\bar{e})=\prod_{i=1}^{m}\left(\sin \rho+a_{i}(e) \cos \rho\right)$. Hence, denoting by $d \sigma^{l}$ the standard volume element of $S^{l}$,

$$
\begin{aligned}
\tau(\imath \cdot f, x) & =\left(c_{m+k}\right)^{-1} \int_{N_{\imath \cdot f}^{1}(x)}|G(e)| d \sigma^{k}(\bar{e}) \\
& =\left(c_{m+k}\right)^{-1} \int_{-\pi / 2}^{\pi / 2} \int_{N_{f}^{1}(x)} \prod_{\imath=1}^{m}\left|\sin \rho+a_{\imath}(e) \cos \rho\right| \cos ^{k-1} \rho d \rho \wedge d \sigma^{k-1}(e)
\end{aligned}
$$


Now we consider the absolute curvature of $\pi_{p} \cdot f$. For $p \in S^{m+k}-f(M)$, define a mapping $\lambda_{p}: N_{f}^{1}(x) \rightarrow N_{\pi_{p} \cdot f}^{1}(x)$ by $\lambda_{p}(e):=1 / k\left(\pi_{p}\right)_{*}(e) \quad\left(e \in N_{f}^{1}(x)\right)$. Let $\tilde{e}:=\lambda_{p}(e)$ and let $\tilde{S}_{p}(\tilde{e})$ be the second fundamental tensor of $\pi_{p} \cdot f$ with respect to $\tilde{e}$. Then we have the following lemma:

LEMMA 3. $1 / k\left(X_{i}(e)\right)$ are the principal vectors of $\pi_{p} \cdot f$ with respect to $\tilde{e}=$ $\lambda_{p}(e)$ with the principal curvatures $1 / k\left(a_{i}(e)+k / 2\langle e, p\rangle\right)$ respectively.

Proof. In this proof we identify $M$ with its image by $f$. Let $\alpha(t)$ be a $C^{1}$-curve in $M$ with $\alpha(0)=x$ and $\alpha^{\prime}(0)=X_{\imath}(e)$. Take $e(t)=e(\alpha(t))$, a normal vector field of $f$ along $\alpha(t)$, such that $e(0)=e$. Let ()$^{T}: T_{\pi_{p} \cdot f(x)} E^{m+k} \rightarrow T_{x} M$ be the tangential projection of $\pi_{p} \cdot f$. Since

$$
\tilde{S}_{p}(\tilde{e})\left(\frac{1}{k} X_{\imath}(e)\right)=\frac{1}{k}\left(\left.\frac{d}{d t} \tilde{e}(t)\right|_{t=0}\right)^{T}
$$

where $\tilde{e}(t)=\lambda_{\alpha(t)} e(i)$, denoting

$$
\begin{gathered}
k(\alpha(t))=\frac{2}{1-\langle\alpha(t), p\rangle} \quad \text { by } k(t), \\
\tilde{S}_{p}(\tilde{e})\left(\frac{1}{k} X_{i}(e)\right)=\frac{1}{k}\left(\left.\frac{d}{d t}\left(e(t)+\frac{k(t)}{2}\langle e(t), p\rangle(\alpha(t)-p)\right)\right|_{t=0}\right)^{T} \\
=\frac{1}{k}\left(e^{\prime}(0)+\frac{k^{\prime}(0)}{2}\langle e, p\rangle(x-p)+\frac{k}{2}\left\langle e^{\prime}(0), p\right\rangle(x-p)\right. \\
\left.+\frac{k}{2}\langle e, p\rangle X_{\imath}(e)\right)^{T}
\end{gathered}
$$

Since $\left(\left(\pi_{p}\right)_{*}\left(e^{\prime}(0)\right)\right)^{T}=S_{e}\left(X_{i}(e)\right)=a_{i}(e) X_{i}(e)$,

$$
\begin{aligned}
\tilde{S}_{p}(\tilde{e})\left(\frac{1}{k} X_{\imath}(e)\right)= & \frac{1}{k}\left(\frac{1}{k}\left(\pi_{p}\right)_{*}\left(e^{\prime}(0)\right)\right. \\
& \left.+\frac{k}{2}\langle e, p\rangle\left(\pi_{p}\right)_{*}\left(\frac{1}{k} X_{i}(e)\right)\right)^{T} \\
= & \frac{1}{k}\left(a_{i}(e)+\frac{k}{2}\langle e, p\rangle\right)\left(\frac{1}{k} X_{\imath}(e)\right)
\end{aligned}
$$

q. e. d.

Let $\tilde{G}_{p}(\tilde{e})$ be the Lipschitz-Killing curvature of $\pi_{p} \cdot f$ with respect to $\tilde{e}=$ $\lambda_{p}(e) \in N_{\pi_{p} \cdot f}^{1}(x)$. Since $\tilde{G}_{p}(\tilde{e})=\prod_{i=1}^{m}\left(a_{i}(e)+k / 2\langle e, p\rangle\right)$ and $\lambda_{p}$ is an isometry of $N_{f}^{1}(x)$ into $N_{\pi_{p} \cdot f}^{1}(x)$, 


$$
\begin{aligned}
\tau\left(\pi_{p} \cdot f, x\right) & =\left(c_{m+k-1}\right)^{-1} \int_{N_{\pi_{p} \cdot f^{(x)}}^{1}}\left|\tilde{G}_{p}(\tilde{e})\right| d \sigma^{k-1}(\tilde{e}) \\
& =\left(c_{m+k-1}\right)^{-1} \int_{N_{f}^{1}(x)} \frac{1}{k^{m}} \prod_{\imath=1}^{m}\left|a_{\imath}(e)+\frac{k}{2}\langle e, p\rangle\right| d \sigma^{k-1}(e)
\end{aligned}
$$

Proof of Theorem.

We now assume that $M$ is orientable. When $M$ is non-orientable, the proof of the theorem can be reduced to the orientable case through the double covering of $M$.

Let $d V$ be the volume element of $M$ with respect to the metric induced from $E^{m+k+1}$ by $\imath \cdot f$ and $d V_{p}$ be the one with respect to the metric induced from $E^{m+k}$ by $\pi_{p} \cdot f$. Then we have $d V_{p}=k^{m} d V$. Since

$$
\tau(\imath \cdot f)=\int_{M} \tau(\imath \cdot f, x) d V(x) \text { and } \tau\left(\pi_{p} \cdot f\right)=\int_{M} \tau\left(\pi_{p} \cdot f, x\right) d V_{p}(x),
$$

by (1) and (2), it is sufficient for the proof of the theorem to show the following equality (3) for any $x \in M$ and $e \in N_{f}^{1}(x)$ :

$$
\begin{aligned}
& \int_{-\pi / 2}^{\pi / 2} \prod_{\imath=1}^{m}\left|\sin \rho+a_{i}(e) \cos \rho\right| \cos ^{k-1} \rho d \rho \\
& \quad=\left(c_{m+k-1}\right)^{-1} \int_{S^{m+k}} \prod_{\imath=1}^{m}\left|a_{\imath}(e)+\frac{k}{2}\langle e, p\rangle\right| d \sigma^{m+k}(p)
\end{aligned}
$$

We parameterize $S^{m+k}$ by $p=\left(\sin ^{2} \theta_{1}-\cos ^{2} \theta_{1} \sin \theta_{2}, \sin \theta_{1} \cos \theta_{1}\left(1+\sin \theta_{2}\right), \cos \theta_{1}\right.$ $\cos \theta_{2} \sin \theta_{3}, \cos \theta_{1} \cos \theta_{2} \cos \theta_{3} \sin \theta_{4}, \cdots \cdots, \cos \theta_{1} \cos \theta_{2} \cdots \cdots \cos \theta_{m+k-1} \sin \theta_{m+k}, \cos \theta_{1}$ $\left.\cos \theta_{2} \cdots \cdots \cdot \cos \theta_{m+k-1} \cos m_{m+k}\right)\left(-\pi / 2 \leqq \theta_{1}, \cdots \cdots, \theta_{m+k-1} \leqq \pi / 2,-\pi \leqq \theta_{m+k} \leqq \pi\right)$.

The volume element of $S^{m+k}$ in terms of this coordinate system is

$$
\begin{aligned}
d \sigma^{m+k}(p)=\cos ^{m+k-1} \theta_{1}\left(1+\sin \theta_{2}\right) \cos ^{m+k-2} \theta_{2} \cos ^{m+k-3} \theta_{3} \cdots \cdots & \\
\cdots \cdots \cdot \cos \theta_{m+k-1} d \theta_{1} \wedge d \theta_{2} \cdots \cdots & \wedge d \theta_{m+k-1} \wedge d \theta_{m+k}
\end{aligned}
$$

We may assume $x=(1,0, \cdots \cdots, 0)$ and $e=(0,1,0, \cdots \cdots, 0)$. Then $k / 2\langle e, p\rangle$ $=\tan \theta_{1}$. Hence

$$
\begin{aligned}
& \int_{S m+k} \prod_{\imath=1}^{m}\left|a_{\imath}(e)+\frac{k}{2}\langle e, p\rangle\right| d \sigma^{m+k}(p) \\
& =\int_{-\pi / 2}^{\pi / 2} \cdots \cdots \cdot \int_{-\pi / 2}^{\pi / 2} \int_{-\pi}^{\pi} \prod_{\imath=1}^{m}\left|a_{\imath}(e)+\tan \theta_{1}\right| \cos ^{m+k-1} \theta_{1}\left(1+\sin \theta_{2}\right) \\
& \quad \times \cos ^{m+k-2} \theta_{2} \cos ^{m+k-3} \theta_{3} \cdots \cdots \cos \theta_{m+k-1} d \theta_{1} d \theta_{2} \cdots \cdots d \theta_{m+k}
\end{aligned}
$$




$$
\begin{aligned}
& =c_{m+k-1} \int_{-\pi / 2}^{\pi / 2} \prod_{\imath=1}^{m}\left|a_{\imath}(e)+\tan \theta_{1}\right| \cos ^{m+k-1} \theta_{1} d \theta_{1} \\
& =c_{m+k-1} \int_{-\pi / 2}^{\pi / 2} \prod_{\imath=1}^{m}\left|\sin \theta_{1}+a_{\imath}(e) \cos \theta_{1}\right| \cos ^{k-1} \theta_{1} d \theta_{1}
\end{aligned}
$$

Thus the equality (3) is shown and the proof of the theorem is completed.

\section{REFERENCES}

[1] T.F. Banchoff, Total central curvature of curves, Duke Math. J., 37 (1970) 281-289

[2] D.Ferus, Totale Absolutkrümmung in Differentialgeometrie und -topologie, Lecture Notes in Mathematics No.66, Springer-Verlag (1968).

Tokyo Institute of Technology 